\title{
Suspension rheology of adhesive particles at high shear-rates
}

\author{
J. J. J. Gillissen $\odot,{ }^{1,}{ }^{*}$ A. Papadopoulou, ${ }^{2}$ S. Balabani, ${ }^{2}$ M. K. Tiwari, ${ }^{2,3}$ and H. J. Wilson ${ }^{1}$ \\ ${ }^{1}$ Department of Mathematics, University College London, London WC1E 6BT, United Kingdom \\ ${ }^{2}$ Department of Mechanical Engineering, University College London, London WC1E 6BT, United Kingdom \\ ${ }^{3}$ Wellcome/EPSRC Centre for Interventional and Surgical Sciences, University College London, \\ London WC1E 6BT, United Kingdom
}

(Received 26 February 2019; accepted 14 April 2020; published 22 May 2020)

\begin{abstract}
A scaling relationship is developed for the asymptotic approach of the suspension viscosity of adhesive, rigid, and non-Brownian particles to that of nonadhesive particles in the limit of high shear-rate where the agglomerates are broken down completely. The relationship is fitted to experimental data for spherical particles, irregularly shaped particles, and biconcave, discoid-shaped, hardened red blood cells. The relationship allows us to extract a proxy for the interparticle adhesion force, which, for the case of the blood cells, is in qualitative agreement with alternative force measurements in the literature.
\end{abstract}

DOI: 10.1103/PhysRevFluids.5.053302

\section{INTRODUCTION}

It is well known that an adhesive interparticle force $F$ induces the formation of particle agglomerates [1,2]. At small applied stress, these structures may span the entire domain, and the material behaves as an elastic solid. When the applied stress exceeds a threshold value, referred to as the yield stress $\Sigma^{*}$, the solid disintegrates and the agglomerates continuously break and regenerate, which corresponds to a viscoelastic fluid. With increasing shear-rate $\dot{\gamma}$, the average agglomerate size decreases and eventually the agglomerates are completely broken down into the primary, particles of size $a$.

In the literature, the yield stress $\Sigma^{*}$ has been related to the interaction force $F$ as $[3,4]$

$$
\Sigma^{*} \sim \phi^{2} \frac{F}{a^{2}},
$$

and similar relations have been postulated that relate $F$ to the elastic modulus [4,5]. These relations are based on the notion that the yield stress is proportional to the interparticle contact number density, which in Eq. (1) is assumed to depend quadratically on the particle volume fraction $\phi$. This scaling assumption is questionable, however, due to the fractal shape of the agglomerates: the number of contacts in a fractal does not necessarily scale as $\phi^{2}$. Because of this complexity, there is no single universally valid scaling relationship between the yield stress $\Sigma^{*}$ and the adhesion force $F$.

In the limit of high $\dot{\gamma}$, however, we will show that there is a universal scaling relationship between the shear stress and the adhesion force $F$. In this limit, the agglomerates are broken down into the primary particles, and the effect of the interactions on the rheology is independent of the structural properties of the agglomerates. In this limit, the effect of the interactions is therefore governed by the microstructure of the nonaggregating particle suspensions, which is independent of the adhesion. When the interactions are assumed short-ranged and the volume fraction sufficiently low, the adhesive interactions act between pairs of primary particles. These conditions imply a universal

*jurriaangillissen@gmail.com 
scaling relationship for the suspension viscosity at high $\dot{\gamma}$. This scaling relationship allows us to extract a proxy for the adhesion force $F$ from rheological data.

\section{SCALING RELATIONSHIP}

\section{A. Proposed scaling relationship}

We consider the nonhydrodynamic part of the relative viscosity in steady shear, which is sometimes referred to as the virial contribution to the relative viscosity or the "virial viscosity" for short,

$$
\Delta \eta_{r}=\eta_{r}-\eta_{r, \infty}
$$

Here $\eta_{r}=\Sigma /(\dot{\gamma} \eta)$ is the suspension relative viscosity, $\Sigma$ is the suspension shear stress, $\eta$ is the dynamic viscosity of the suspending medium, and $\eta_{r, \infty}$ is referred to as the "infinite viscosity" and denotes the value of $\eta_{r}$ at high $\dot{\gamma}$, i.e., where the effect of the adhesion force $F$ is negligible. The particles are assumed to be rigid and non-Brownian. It is noted that despite high $\dot{\gamma}$, the flow is assumed to be laminar, i.e., the Reynolds number is assumed to be $\dot{\gamma} H^{2} / \nu \ll 1$. Here $H$ is the gap height of the rheometer, $v=\eta / \rho$ is the kinematic viscosity of the suspending medium, and $\rho$ is the mass density of the suspending medium. Since in the limits of high $\dot{\gamma}$ and low $\phi$ the adhesive interactions occur between pairs of primary particles, the virial viscosity must scale as $\Delta \eta_{r} \sim \phi^{2}$. We assume a small-particle Reynolds number $\dot{\gamma} a^{2} / v \ll 1$ and a large Péclet number Pe $\gg 1$ :

$$
\mathrm{Pe}=\frac{6 \pi \dot{\gamma} a^{3} \eta}{k_{B} T} .
$$

Under these assumptions, $\Delta \eta_{r}$ is independent of the mass density $\rho$ and of the temperature $k_{B} T$, but it only depends on $F, a, \phi, \dot{\gamma}$, and $\eta$. Dimensional analysis then shows that $\Delta \eta_{r}=$ $\phi^{2} \Psi\left[F /\left(\eta \dot{\gamma} a^{2}\right)\right]$, where $\Psi[x]$ is a nondimensional function of $x$. We make the assumption, which will be experimentally verified below, that $\Psi[x]$ is a power-law function, i.e., $\Psi[x]=C x^{\delta}$ :

$$
\Delta \eta_{r}=C \phi^{2}\left(\frac{F}{\eta \dot{\gamma} a^{2}}\right)^{\delta},
$$

where $\delta$ is an unknown exponent and $C$ is an unknown prefactor of order 1 .

It is noted that $\delta$ and $C$ are unknown functions of particle shape, and these functions cannot be predicted from scaling arguments. Below, the effects of shape are studied by fitting Eq. (4) to experimental data for suspensions of spherical particles, irregularly shaped particles, and biconcave, discoid-shaped, hardened red blood cells.

It is worth reemphasizing that Eq. (4) is based on the assumptions of high $\dot{\gamma}$ and low $\phi$ such that the agglomerates are broken down into the primary particles, and the primary particles interact pairwise. The adhesive interaction depends on the relative positioning of the particles, and, for high $\dot{\gamma}$, this microstructure asymptotes toward that for nonadhesive, i.e., shear-rate invariant suspensions.

\section{B. Preexisting scaling relationships}

Our scaling relationship [Eq. (4)] is different from scaling relationships found in the existing literature. For large $\dot{\gamma}$ and small $\phi$, Ref. [6] theoretically predicts that

$$
\Delta \eta_{r} \sim \phi\left(\frac{F}{\dot{\gamma} \eta a^{2}}\right)^{\delta} .
$$

This theoretical relationship is based on the idea of a shear-rate-dependent effective volume fraction of fractal-shaped agglomerates, and the exponent $\delta$ is argued to depend on the rigidity of the agglomerates. For completeness, we reproduce the derivation leading to Eq. (5) in the Supplemental Material [7]. The scaling $\Delta \eta_{r} \sim \phi$ [Eq. (5)] disagrees with our scaling $\Delta \eta_{r} \sim \phi^{2}$ [Eq. (4)], which is supported by experimental observations (Figs. 3, 4, and 5 below) and which is based on the assumption that adhesive forces act between particle pairs. 
In Ref. [8], discrete element method simulations are conducted of Brownian, adhesive particles. Their numerical method includes thermodynamics forces and long-ranged hydrodynamic interactions, but it ignores short-ranged lubrication forces. Their results follow Eq. (5) with $\delta=0.5$ for relatively strong Brownian motion with Pe $\lesssim 10$ [Eq. (3)], and $\delta=1$ for relatively weak Brownian motion with $\mathrm{Pe} \gtrsim 10$. For completeness, we reproduce these data in the Supplemental Material [7]. The scaling $\Delta \eta_{r} \sim \phi \dot{\gamma}^{-0.5}$ suggests that, under these simulated conditions, the virial viscosity is dominated by interactions between (fractal-shaped) agglomerates, as described by the model [Eq. (5)] of Ref. [6]. The scaling $\Delta \eta_{r} \sim \phi \dot{\gamma}^{-1}$ disagrees with experiments using Brownian particles with $10^{-2} \lesssim \mathrm{Pe} \lesssim 10^{2}$ [3]. This suggests that the observed scaling $\Delta \eta_{r} \sim \phi \dot{\gamma}^{-1}$ is due to the neglect of lubrication forces. In this regard, it is noted that, although lubrication forces have a negligible effect on $\eta_{r}$ at vanishing $\phi$, they are essential for $\Delta \eta_{r}$ since $\Delta \eta_{r}$ probes viscosity perturbations due to adhesion forces between closely separated particles.

\section{EXPERIMENTAL DATA}

\section{A. Adhesion proxy}

The validity of Eq. (4) is verified by fitting it to experimental data. The fitting involves determining $\eta_{r, \infty}$ and $\delta$ by minimizing the sum of the squared distance between the measured $\left[\log _{10}(\dot{\gamma}), \log _{10}\left(\eta_{r}-\eta_{r, \infty}\right)\right]$ and a single straight line with a slope of $-\delta$ for various $\phi$. Assuming a relative uncertainty in $\eta_{r}$ of $\epsilon_{\eta_{r}}$, the virial viscosity $\Delta \eta_{r}$ has a relative uncertainty $\epsilon_{\Delta \eta_{r}}$ of

$$
\epsilon_{\Delta \eta_{r}}=\epsilon_{\eta_{r}} \frac{\eta_{r}}{\Delta \eta_{r}}
$$

From Eq. (4) we see that the interparticle adhesion force $F$ is given by

$$
F=C^{-1 / \delta} \eta \dot{\gamma} a^{2} \Delta \eta_{r}^{1 / \delta} \phi^{-2 / \delta} .
$$

Since the prefactor $C$ cannot be determined from the scaling analysis, it is assumed that $C=1$, and under this assumption Eq. (7a) is referred to as the "adhesion proxy" $F^{\prime}$ :

$$
F^{\prime}=\eta \dot{\gamma} a^{2} \Delta \eta_{r}^{1 / \delta} \phi^{-2 / \delta},
$$

which is an order of magnitude estimate of $F$. The relative uncertainty $\epsilon_{F^{\prime}}$ in the adhesion proxy $F^{\prime}$ due to the relative uncertainty $\epsilon_{\Delta \eta_{r}}$ in $\Delta \eta_{r}$ reads

$$
\epsilon_{F^{\prime}}=\delta^{-1} \epsilon_{\Delta \eta_{r}} .
$$

Note that this equation only accounts for uncertainties in $\Delta \eta_{r}$, but not in $\phi$ or $a$.

\section{B. Spherical and irregularly shaped particles}

First we focus on spherical silica particles and on irregularly shaped precipitated silica particles, both suspended in mineral oil, with a viscosity of $\eta=3.1 \times 10^{-2} \mathrm{~Pa}$ s. The interaction forces between the hydrophilic silica particles are mediated by hydrogen bonding between their surface silanol $(\mathrm{OH})$ groups [9]. When suspended in a nonpolar liquid with no hydrogen-bonding capacity, such as mineral oil, the interactions between the silica particles become attractive and the system gels at small shear stress. The silica-mineral oil gel is used in various technologies, including lubricating greases, heat transfer fluids, and filling compounds in fiber-optic cables [9].

The size distributions of the spherical and irregularly shaped particles are measured using laser diffraction, and they are shown in Fig. 1(a). The spherical and the irregularly shaped particles have a somewhat similar mean and standard deviation in the radius of $a=(6 \pm 4)$ and $(8 \pm 7) \mu \mathrm{m}$, respectively. Particle morphologies are shown in scanning electron microscope (SEM) images in Figs. 1(b) and 1(c).

The sheared suspension is visualized in an optical shearing cell, i.e., in the $100 \mu \mathrm{m}$ gap between one steady and one rotating quartz plate (CSS-450, Linkam Scientific). Prior to imaging at a certain 

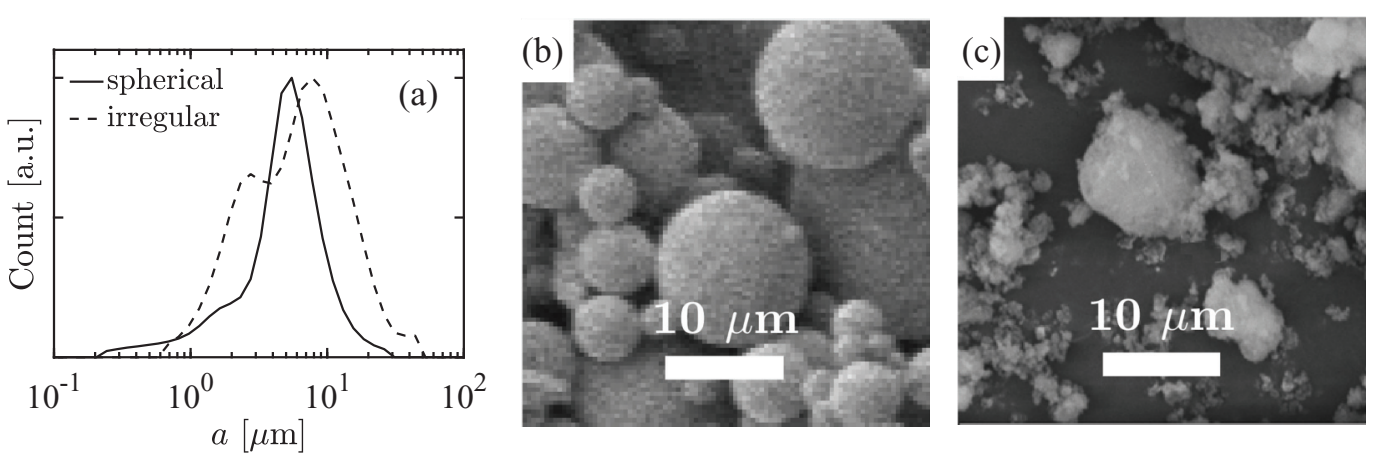

FIG. 1. (a) Particle radius distribution measured by laser diffraction for spherical particles and irregularly shaped particles. (b) SEM image of spherical particles. (c) SEM image of irregularly shaped particles.

desired shear-rate, the suspension is sheared for $2 \mathrm{~min}$ at $1000 \mathrm{~s}^{-1}$ to disintegrate the agglomerates. Thereafter, the suspension is sheared at the desired shear-rate for $10 \mathrm{~min}$ to reach fully developed agglomerate statistics. After these preshearing steps, the suspension is sheared at the desired shearrate, illuminated using a fiber optic light emitting diode (SugarCube Ultra, Edmund Optics), and imaged using a high-speed camera (IDT XS-3 CMOS) with a resolution of $1280 \times 1024$ pixels and a $10 \times$ objective, resulting in $0.5 \mu \mathrm{m} / \mathrm{pixel}$, while the temperature is maintained at $20^{\circ} \mathrm{C}$. Figures 2 (a) and 2(b) show images at low and high shear-rate for the spherical particles, and Figs. 2(c) and 2(d) show images for the irregularly shaped particles. In both systems, agglomerates are observed at low shear-rate and these are broken down into the primary particles at high shear-rate $\left(\dot{\gamma}=500 \mathrm{~s}^{-1}\right)$. It is also noted that the agglomerates at the low shear-rate are larger for the irregularly shaped particles than for the spherical ones. To properly visualize the agglomerates in both systems, Figs. 2(a) and 2(b) and Figs. 2(c) and 2(d) correspond to different image sizes.

For both systems, we study the scaling relationship between the virial viscosity $\Delta \eta_{r}$, the volume fraction $\phi$, and the shear-rate $\dot{\gamma}$. To this end, their shear viscosities are measured as functions of the shear-rate $\dot{\gamma}$ at various volume fractions $\phi$ [Fig. 3(a)]. The measurements are conducted at $20^{\circ} \mathrm{C}$ using a hybrid stress controlled rheometer (DHR-3, TA Instruments), employing a stainless steel parallel plate geometry, sandblasted with a roughness of $2 \mu \mathrm{m}$ and a gap width of $650 \mu \mathrm{m}$. To correct for the heterogeneous shear rate in the parallel plate geometry, we apply the Rabinowitch correction [10]. The relative measurement uncertainty in $\eta_{r}$ (upon repeating the experiment) is $\epsilon_{\eta_{r}} \sim 10^{-2}$. We consider shear-rates between $\dot{\gamma}=10$ and $10^{3} \mathrm{~s}^{-1}$. By comparing upward and downward shear sweeps in the Supplemental Material [7], we confirm that, in this range, the viscosity is independent of the shear history. For both particle suspensions, $\Delta \eta_{r}$ is extracted from the rheological data using the protocol that is described in Sec. III A.

Figure $3(\mathrm{~b})$ shows the resulting infinite viscosity $\eta_{r, \infty}$ where the error bars give the standard deviation of four shear-sweeps (two up-sweeps and two down-sweeps using two different samples). We note that per sweep the uncertainty in $\eta_{r, \infty}$ (obtained by curve fitting) is negligible $(<1 \%)$, and we emphasize that the error bars in Fig. 3(b) represent variability between different sweeps corresponding to different samples. For the spherical particles $\eta_{r, \infty}$ roughly follows the Batchelor relation $\eta_{r, \infty}=1+2.5 \phi+5.2 \phi^{2}$, which confirms the physical significance of $\eta_{r, \infty}$. For the irregularly shaped particles, $\eta_{r, \infty}$ deviates from Batchelor's equation, which may be attributed to nonsphericity and to the uncertainty in $\phi$ of these porous particles [see Fig. 1(c)].

Figure 3(c) shows the resulting virial viscosity $\Delta \eta_{r}$ where the error bars are the corresponding relative uncertainties $\epsilon_{\Delta \eta_{r}}$ [Eq. (6)], which are large for small $\phi$ and large $\dot{\gamma}$. We note that the data 

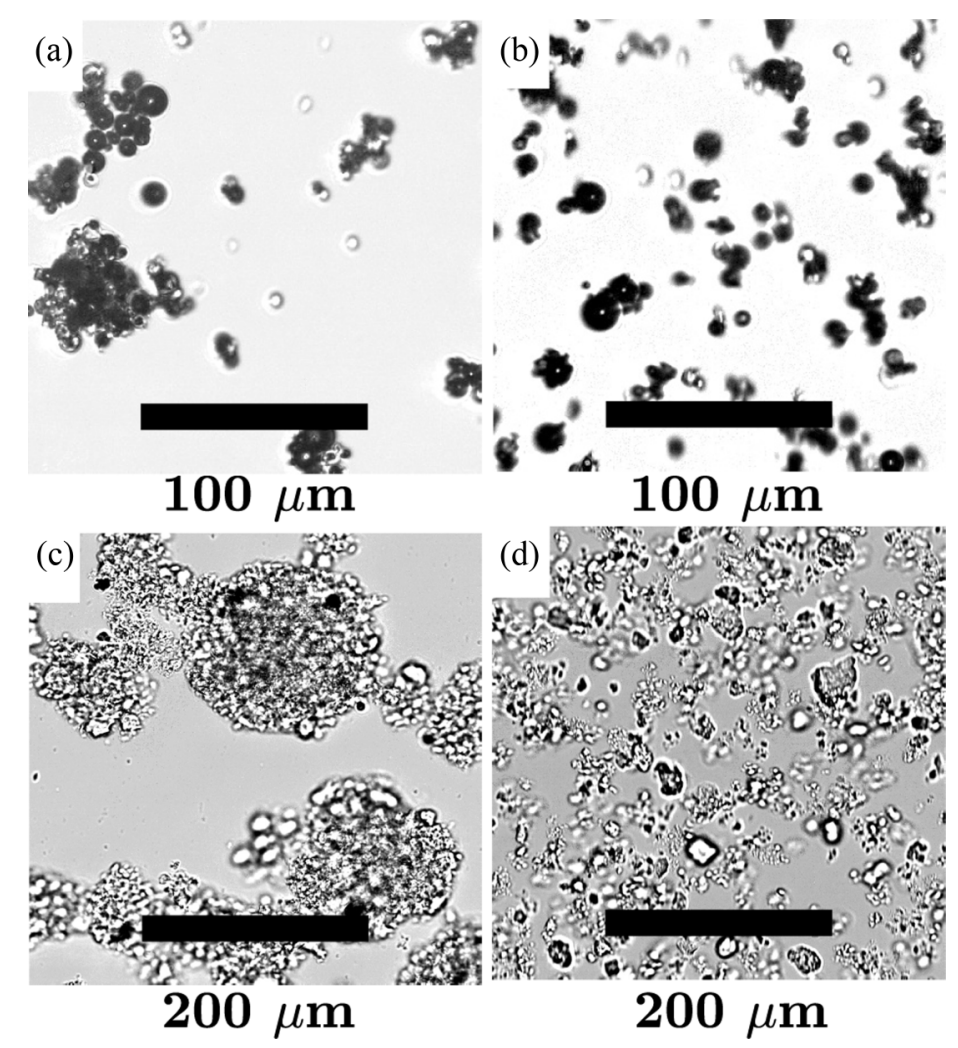

FIG. 2. Photograph of (agglomerates of) spherical silica particles for $\phi=0.01$ in mineral oil, sheared at $\dot{\gamma}=50 \mathrm{~s}^{-1}$ (a) and at $500 \mathrm{~s}^{-1}$ (b) and of irregularly shaped silica particles for $\phi=0.01$ in mineral oil, sheared at $\dot{\gamma}=20 \mathrm{~s}^{-1}$ (c) and at $500 \mathrm{~s}^{-1}$ (d).

in Fig. 3(c) correspond to single sweeps and are found by subtracting from $\eta_{r}$ [Fig. 3(a)] the value for $\eta_{r, \infty}$ for a single sweep. Unlike the large variability in $\eta_{r, \infty}$ upon repeating sweeps [Fig. 3(b)], the uncertainty in a single $\eta_{r, \infty}$ is negligibly small. Therefore, the error in Fig. 3(c) is not affected by the variability in $\eta_{r, \infty}$ [Fig. 3(b)], but it is solely due to the $1 \%$ measurement error in $\eta_{r}$ which is propagated by Eq. (6) into the error of $\Delta \eta_{r}$. The data in Fig. 3(c) are very well fitted over two decades of shear-rate by $\Delta \eta_{r} \sim \dot{\gamma}^{-\delta}$ with $\delta=0.5$ (dashed lines), which confirms the presupposed power-law dependence [Eq. (4)].

In Fig. 3(d) the resulting adhesion proxy $F^{\prime}$ [Eq. (7b) using $\delta=0.5$ ] is plotted as a function of $\dot{\gamma}$ for both particle suspensions and for various $\phi$ where the error bars correspond to Eq. (8). For both suspensions, the data agree well with Eq. (7b) using $\delta=0.5$ (dashed lines). The observed $\Delta \eta_{r} \sim \phi^{2}$ scaling reflects the dominant contribution of pairwise interactions to $\Delta \eta_{r}$, which is expected in the limit of vanishing $\phi$.

From the fit of the horizontal lines in Fig. 3(d), it is found that $F^{\prime}=4.5 \times 10^{-6}$ and $6.0 \times 10^{-5} \mathrm{~N}$ for the spherical particles and for the irregularly shaped particles, respectively. These results confirm that the adhesion force between irregularly shaped particles is larger than that between spherical particles since there are more contact points per particle-particle interaction. It is emphasized that the actual adhesion force $F$ differs from the adhesion proxy $F^{\prime}$ by an unknown dimensionless constant of order 1 which depends on the particle shape. This constant cannot be determined from scaling arguments. 

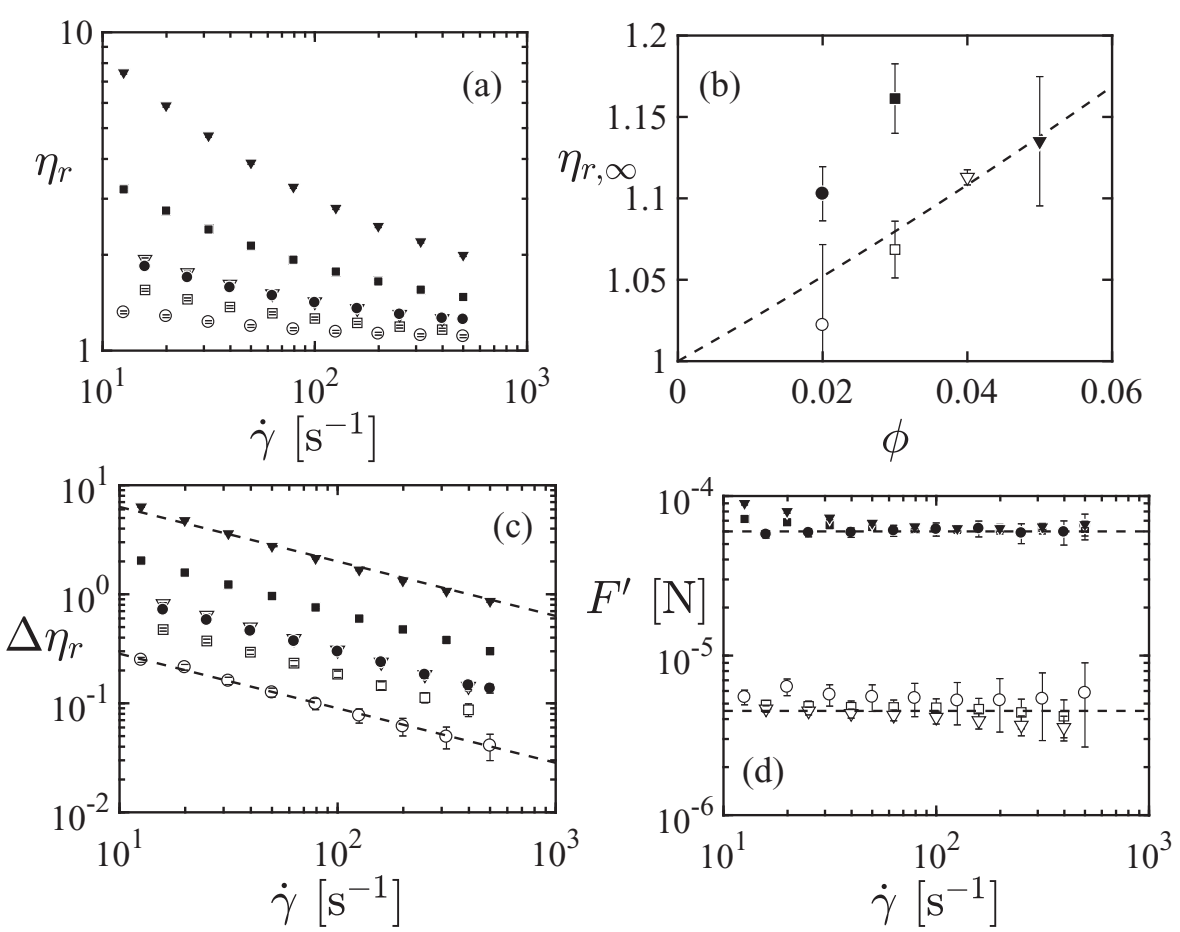

FIG. 3. (a) Relative viscosity, (b) infinite viscosity, (c) virial viscosity, and (d) adhesion proxy $F^{\prime}$ [Eq. (7b) using $\delta=0.5$ ]. The open symbols are the spherical particles for $\phi=0.02(\circ), 0.03(\square), 0.04(\nabla)$ and the filled symbols are the irregularly shaped particles for $\phi=0.02(\bullet), 0.03(\boldsymbol{\nabla}), 0.05(\mathbf{\nabla})$. The error bars in (a) are smaller than the marker sizes. The dashed line in (b) is the Batchelor relation $\eta_{r, \infty}=1+2.5 \phi+5.2 \phi^{2}$. The dashed lines in (c) have slopes of -0.5 and represent Eq. (4) with $\delta=0.5$. The horizontal dashed lines in (d) indicate Eq. (7b) with $\delta=0.5$.

\section{Monodisperse, spherical particles}

Next Eq. (4) is fitted to experimental data from Ref. [11], concerning monodisperse, spherical silica particles in mineral oil with viscosity $\eta=0.12 \mathrm{~Pa}$ s. Two data sets are analyzed, involving spherical particles with diameters of $110 \pm 10 \mathrm{~nm}$ and $21 \pm 3 \mu \mathrm{m}$, which are referred to as "silica $110 \mathrm{~nm}$ " and "silica $21 \mu \mathrm{m}$," respectively. The scaling relationship is studied between the virial viscosity $\Delta \eta_{r}$, the volume fraction $\phi$, and the shear-rate $\dot{\gamma} \cdot \Delta \eta_{r}$ is extracted from the rheological data using the protocol that is described in Sec. III A.

In Fig. 4 the adhesion proxy $F^{\prime}$ [Eq. (7b) using $\delta=0.5$ ] is plotted as a function of the shear rate, for both silica $110 \mathrm{~nm}$ and silica $21 \mu \mathrm{m}$ and for various volume fractions where the error bars correspond to Eq. (8). It is noted that $\eta_{r}$ is extracted from the printed figures in Ref. [11] "by hand" with a (relative) uncertainty $\epsilon_{\eta_{r}} \approx 0.02$, which introduces large (relative) uncertainties in $\Delta \eta_{r}$ [Eq. (6)] and in $F^{\prime}$ [Eq. (8)] at the lower $\phi$ and the higher $\dot{\gamma}$ where $\Delta \eta_{r}$ is small. Also note that, for $\epsilon_{F^{\prime}}>1$ [Eq. (8)], the lower half of the error bars is not displayed.

The collapse in Fig. 4 of $F^{\prime}$ for various $\phi$ at high $\dot{\gamma}$ supports Eq. (4) with an exponent of $\delta=$ 0.5 , i.e., $\Delta \eta_{r} \sim \phi^{2} \dot{\gamma}^{-0.5}$. Surprisingly, the $\Delta \eta_{r} \sim \phi^{2}$ scaling is observed even at relatively high $\phi=0.3$ where multibody, short-ranged interactions could be expected, but apparently these are subdominantly affecting $\Delta \eta_{r}$ in this system. It is further noted that the agreement between the experimental data and Eq. (4) is better for the larger silica $21 \mu \mathrm{m}$ particles than for the smaller silica $110 \mathrm{~nm}$ particles. For the latter there are deviations for low $\dot{\gamma}$ and the data collapse only at high $\dot{\gamma}$. These discrepancies may be attributed to Brownian motion. For silica $110 \mathrm{~nm}$, the Péclet number Pe [Eq. (3)] varies between $7 \times 10^{-1} \leqslant \mathrm{Pe} \leqslant 7 \times 10^{1}$ for $10^{1} \mathrm{~s}^{-1} \leqslant \dot{\gamma} \leqslant 10^{3} \mathrm{~s}^{-1}$. This confirms 


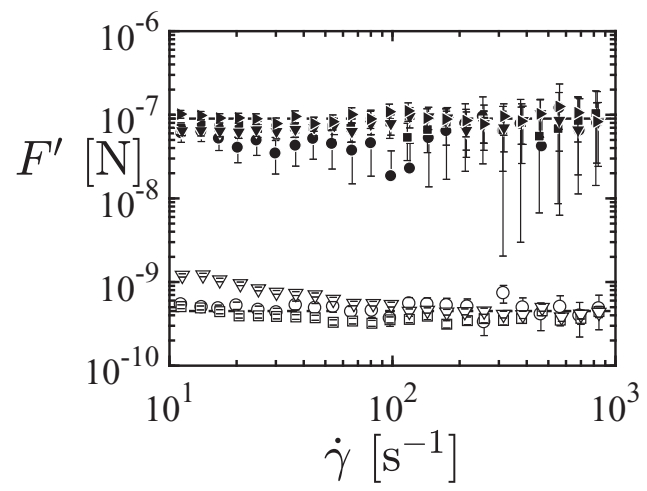

FIG. 4. Adhesion proxy $F^{\prime}$ [Eq. (7b) using $\delta=0.5$ ] as a function of the shear-rate $\dot{\gamma}$ at different particle volume fractions $\phi$. Open symbols are $110 \mathrm{~nm}$ diameter silica spheres in mineral oil for $\phi=0.05(\circ), 0.1(\square)$, and $0.15(\nabla)[11]$. Filled symbols are $21 \mu \mathrm{m}$ diameter silica spheres in mineral oil for $\phi=0.15(\bullet), 0.2(\mathbb{\square})$, $0.25(\mathbf{v})$, and $0.30(\nabla)[11]$.

the hypothesis that the deviations in Fig. 4 from Eq. (4) for silica $110 \mathrm{~nm}$ at the lower shear-rates are due to Brownian motion, while the agreement with Eq. (4) at the higher shear-rates corresponds to negligible Brownian motion. For silica $21 \mu \mathrm{m}$, on the other hand, Brownian motion is negligible over the entire $\dot{\gamma}$-range as Pe varies between $6 \times 10^{6} \leqslant \mathrm{Pe} \leqslant 6 \times 10^{8}$ for $10^{1} \mathrm{~s}^{-1} \leqslant \dot{\gamma} \leqslant 10^{3} \mathrm{~s}^{-1}$.

From Fig. 4(b), it is seen that $F^{\prime}=4.5 \times 10^{-10}$ and $9 \times 10^{-8} \mathrm{~N}$ for silica $110 \mathrm{~nm}$ and silica $21 \mu \mathrm{m}$, respectively. It is reemphasized that the actual adhesion force $F$ differs from the apparent adhesion force $F^{\prime}$ by an unknown dimensionless constant of order one. The relative values of $F^{\prime}$ for silica $110 \mathrm{~nm}$ and for silica $21 \mu \mathrm{m}$, however, support the theoretical relationship $F=2 \pi s a$ between the adhesion force $F$, the particle radius $a$, and the surface energy $s$ [12] which, in this case, is mediated by hydrogen bonding between the surface silanol groups [9].

\section{Red blood cells}

Red blood cells (RBCs) are biconcave discoids with a diameter of approximately $7 \mu \mathrm{m}$, a thickness at the thickest point of approximately $2 \mu \mathrm{m}$, and a minimum thickness in the center of approximately $1 \mu \mathrm{m}$, which correspond to a surface area of $A \approx 136 \mu \mathrm{m}^{2}$, an equivalent radius of $a=\sqrt{A /(4 \pi)} \approx 3 \mu \mathrm{m}$, and an aspect ratio of around 3. The non-Newtonian behavior of blood arises from RBC deformability and aggregation. Aggregation between RBCs is driven by attractive forces that are the result of an osmotic pressure gradient due to the depletion of large plasma proteins, primarily fibrinogen, in the narrow intercellular regions [13].

In the RBC suspension experiment in Ref. [6], cell deformability was inhibited by treating the cells with glutaraldehyde. The hardened cells were suspended in Boger fluids, which consist of aqueous solutions of salt and the neutral polysaccharide dextran that induces adhesive depletion forces. Two different dextran polymers were used with a molecular weight of $M_{w}=75$ and $150 \mathrm{~kg} / \mathrm{mol}$. These systems are referred to as "RBC 75" and "RBC 150," respectively. In both systems, the dextran concentration is fixed at $2 \mathrm{~g} / \mathrm{dL}$.

$\Delta \eta_{r}$ is extracted from the data in Ref. [6] using the protocol that is described in Sec. III A. Figure 5 shows the resulting adhesion proxy $F^{\prime}$ [Eq. (7b)] using $\delta=0.55$ for RBC 75 and $\delta=0.6$ for RBC 150 as a function of $\dot{\gamma}$ for various $\phi$. The viscosity of the dextran solutions is taken to be $\eta \approx 1.4 \times 10^{-3} \mathrm{~Pa}$ s for $\mathrm{RBC} 75$ and $\eta \approx 1.6 \times 10^{-3} \mathrm{~Pa}$ s for RBC 150 [14]. It is noted again that $\eta_{r}$ is extracted from the printed figures in Ref. [6] "by hand" with a (relative) uncertainty $\epsilon_{\eta_{r}} \approx 0.02$, which introduces large (relative) uncertainties in $\Delta \eta_{r}$ [Eq. (6)] and in $F^{\prime}$ [Eq. (8)] at the higher $\dot{\gamma}$ where $\Delta \eta_{r}$ is small. Also note that, for $\epsilon_{F^{\prime}}>1$ [Eq. (8)], the lower half of the error bars is not displayed. 


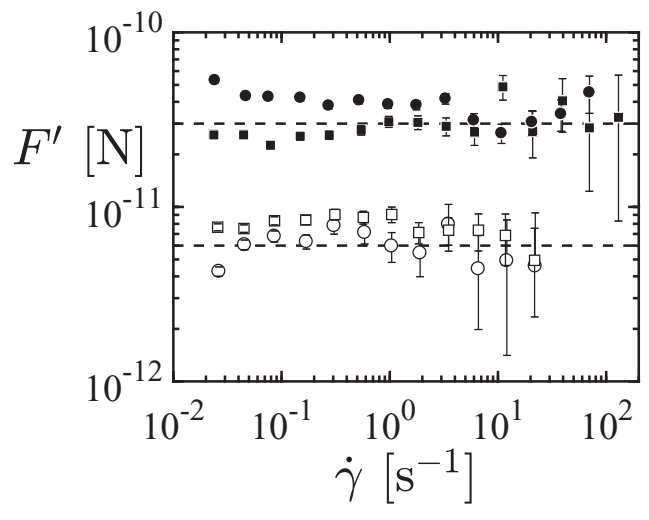

FIG. 5. Adhesion proxy $F^{\prime}$ [Eq. (7b)] using $\delta=0.55$ for RBC 75 (open symbols) and $\delta=0.6$ for RBC 150 (filled symbols) as a function of the shear-rate $\dot{\gamma}$ for different RBC volume fractions $\phi=0.13$ ( $\circ$ and $\bullet$ ) and 0.21 ( $\square$ and $\mathbf{\square})$ [6].

The larger exponents $\delta=0.55$ for $\mathrm{RBC} 75$ and $\delta=0.6$ for $\mathrm{RBC} 150$ than $\delta=0.5$ for spheres might indicate a shape effect. The irregular particles in Fig. 3(d) (filled symbols), however, have a "sphere-like exponent" $\delta=0.5$. Moreover, the dependence of $\delta$ on the polymer weight suggests that the larger exponent is due to the non-Newtonian rheology of the suspending medium. Although the dextran solutions are Boger fluids, the rheology in the squeeze films between the RBCs may be non-Newtonian, due to extensional flow topologies.

From the fit of the horizontal lines in Fig. 5, it is found that $F^{\prime}=6 \times 10^{-12}$ and $3 \times 10^{-11} \mathrm{~N}$ for RBC 75 and RBC 150, respectively. These results are compared to the force measurements of Ref. [15]. These authors measured the force that is required to separate two deformable RBCs where one is attached to a flat surface and the other is attached to the cantilever of an atomic force microscope (AFM). The suspending medium is a solution of salt and dextran with molecular weights of $M_{w}=70$ and $150 \mathrm{~kg} / \mathrm{mol}$, which are very close to the values used in [6]. At a dextran concentration of $2 \mathrm{~g} / \mathrm{dL}$, the measured adhesion forces are $F=6 \times 10^{-11}$ and $1.5 \times 10^{-10} \mathrm{~N}$ for $M_{w}=70$ and $150 \mathrm{~kg} / \mathrm{mol}$, which are ten and five times as large as our findings.

These discrepancies are attributed to the unknown dimensionless constant $C^{-1 / \delta}$ in the definition of the adhesion force $F$ in Eq. (7a) as well as to differences in cell treatment, resulting in different surface chemistry and membrane deformability. The cells of Ref. [15] interact via deformable contact surfaces, while in Ref. [6] the hardened cells interact via contact points. Despite these sources of uncertainty and differences in material properties, which are inherent when dealing with biological samples, both results are in the same range, which supports the validity of Eq. (4).

\section{CONCLUSION}

The scaling relationship is studied between the virial viscosity $\Delta \eta_{r}=\eta_{r}-\eta_{r, \infty}$, the shear-rate $\dot{\gamma}$, and the particle volume fraction $\phi$ in the limits of high $\dot{\gamma}$ and low $\phi$. Here $\eta_{r}$ is the relative suspension viscosity and $\eta_{r, \infty}$ denotes the value of $\eta_{r}$ at high $\dot{\gamma}$ (while remaining laminar), which when subtracted from $\eta_{r}$ gives a power law: $\Delta \eta_{r} \sim \phi^{2} \dot{\gamma}^{-\delta}$ at high $\dot{\gamma}$, i.e., a straight line on $\left[\log _{10}(\dot{\gamma}), \log _{10}\left(\Delta \eta_{r}\right)\right]$ coordinates. The scaling relationship is fitted to experimental data for spherical particles, irregularly shaped particles, and biconcave, discoid-shaped, hardened red blood cells. The relationship allows us to extract a proxy for the interparticle adhesion force that, for the case of the blood cells, is in qualitative agreement with alternative force measurements in the literature. 


\section{ACKNOWLEDGMENTS}

We would like to acknowledge financial support from the Engineering and Physical Sciences Research Council of the United Kingdom, Grant No. EP/N024915/1. We thank Glaxo Smith Kline for donating the irregularly shaped silica particles and the corresponding SEM images. We also thank Microtrac for conducting the laser diffraction particle size measurements.

[1] W. B. Russel, D. A. Saville, and W. R. Schowalter, Colloidal Dispersions (Cambridge University Press, Cambridge, 1991).

[2] J. Mewis and N. J. Wagner, Colloidal Suspension Rheology (Cambridge University Press, Cambridge, 2012).

[3] A. P. R. Eberle, N. Martys, L. Porcar, S. R. Kline, W. L. George, J. M. Kim, P. D. Butler, and N. J. Wagner, Shear viscosity and structural scalings in model adhesive hard-sphere gels, Phys. Rev. E 89, 050302(R) (2014).

[4] J. M. Kim, A. P. R. Eberle, A. K. Gurnon, L. Porcar, and N. J. Wagner, The microstructure and rheology of a model, thixotropic nanoparticle gel under steady shear and large amplitude oscillatory shear (LAOS), J. Rheol. 58, 1301 (2014).

[5] M. Clarion, M. Deegan, T. Helton, J. Hudgins, N. Monteferrante, E. Ousley, and M. Armstrong, Contemporary modeling and analysis of steady state and transient human blood rheology, Rheol. Acta 57, 141 (2018).

[6] P. Snabre and P. Mills, I. Rheology of weakly flocculated suspensions of rigid particles, J. Phys. III (France) 6, 1811 (1996).

[7] See Supplemental Material at http://link.aps.org/supplemental/10.1103/PhysRevFluids.5.053302 for a reproduction of the derivation leading to the scaling relationship of Ref. [6], a reproduction of the discrete element simulation data of Ref. [8], and a comparison of our measured virial viscosity during an upward and a downward shear sweep.

[8] Z. Varga and J. W. Swan, Large scale anisotropies in sheared colloidal gels, J. Rheol. 62, 405 (2018).

[9] S. A. Khan and N. J. Zoeller, Dynamic rheological behavior of flocculated fumed silica suspensions, J. Rheol. 37, 1225 (1993).

[10] C. Gamonpilas, J. F. Morris, and M. M. Denn, Shear and normal stress measurements in non-Brownian monodisperse and bidisperse suspensions, J. Rheol. 60, 289 (2016).

[11] S. Chen, G. Øye, and J. Sjöblom, Rheological properties of silica particle suspensions in mineral oil, J. Dispersion Sci. Technol. 26, 791 (2005).

[12] R. S. Bradley, The cohesive force between solid surfaces and the surface energy of solids, Philos. Mag. 13, 853 (1932).

[13] B. Neu and H. J. Meiselman, Depletion-mediated red blood cell aggregation in polymer solutions, Biophys. J. 83, 2482 (2002).

[14] V. Tirtaatmadja, D. E. Dunstan, and D. V. Boger, Rheology of dextran solutions, J. Non-Newtonian Fluid Mech. 97, 295 (2001).

[15] P. Steffen, C. Verdier, and C. Wagner, Quantification of Depletion-Induced Adhesion of Red Blood Cells, Phys. Rev. Lett. 110, 018102 (2013). 